\title{
Prevalence and factors associated with type 2 diabetes mellitus and hypertension among the hill tribe elderly populations in northern Thailand
}

Tawatchai Apidechkul 1,2

\begin{abstract}
Background: Type 2 diabetes mellitus (T2DM) and hypertension (HT) are major noncommunicable health problems in both developing and developed countries, including Thailand. Each year, a large amount of money is budgeted for treatment and care. Hill tribe people are a marginalized population in Thailand, and members of its elderly population are vulnerable to health problems due to language barriers, lifestyles, and daily dietary intake.

Methods: An analytic cross-sectional study was conducted to estimate the prevalence of T2DM and HT and to assess the factors associated with T2DM and HT. The study populations were hill tribe elderly adults aged $\geq 60$ years living in Chiang Rai Province, Thailand. A simple random method was used to select the targeted hill tribe villages and participants into the study. A validated questionnaire, physical examination form, and 5-mL blood specimen were used as research instruments. Fasting plasma glucose and blood pressure were examined and used as outcome measurements. Chi-square tests and logistic regression were used for detecting the associations between variables at the significance level alpha $=0.05$.

Results: In total, 793 participants participated in the study; 49.6\% were male, and 51.7\% were aged 60-69 years. A total of $71.5 \%$ were Buddhist, $93.8 \%$ were uneducated, $62.9 \%$ were unemployed, and $89 \%$ earned an income of $<5,000 \mathrm{baht} / \mathrm{month}$. The overall prevalence of T2DM and HT was $16.8 \%$ and $45.5 \%$, respectively. Approximately 9.0\% individuals had comorbidity of T2DM and HT. Members of the Lahu, Yao, Karen, and Lisu tribes had a greater odds of developing T2DM than did those of the Akha tribe. Being overweight, having a parental history of T2DM, and having high cholesterol were associated with T2DM development. In contrast, those who engaged in highly physical activities and exercise had lower odds of developing T2DM than did those who did not. Regarding HT, being female, having a high dietary salt intake, being overweight, and having a parental history of HT were associated with HT development among the hill tribe elderly populations.

Conclusions: The prevalence of T2DH and HT among the hill tribe elderly populations is higher than that among the general Thai population. Public health interventions should focus on encouraging physical activity and reducing personal weight, dietary salt intake, and greasy food consumption among the hill tribe elderly.
\end{abstract}

Keywords: Type 2 diabetes mellitus, Hypertension, Hill tribe, Elderly, Thailand

Correspondence: tk2016ms@gmail.com; tawatchai.api@mfu.ac.th

${ }^{1}$ Center of Excellence for the Hill tribe Health Research, Mae Fah Luang

University, Chiang Rai, Thailand

${ }^{2}$ School of Health Science, Mae Fah Luang University, Chiang Rai, Thailand

(c) The Author(s). 2018 Open Access This article is distributed under the terms of the Creative Commons Attribution 4.0 International License (http://creativecommons.org/licenses/by/4.0/), which permits unrestricted use, distribution, and reproduction in any medium, provided you give appropriate credit to the original author(s) and the source, provide a link to the Creative Commons license, and indicate if changes were made. The Creative Commons Public Domain Dedication waiver (http://creativecommons.org/publicdomain/zero/1.0/) applies to the data made available in this article, unless otherwise stated. 


\section{Background}

Type 2 diabetes mellitus (T2DM) and hypertension (HT) are common noncommunicable diseases among elderly adults aged $\geq 60$ years in both developing and developed countries [1]. The prevalence of T2DM and HT varies according to age, sex, and race [2, 3]. There are different factors associated with T2DM and HT in different populations, particularly among those with different lifestyles and cultures [3, 4]. Older populations are the most vulnerable to the development of T2DM and HT $[5,6]$. T2DM and HT have become major causes of morbidity and mortality of elderly populations in all countries $[7,8]$. The impact of T2DM and HT is not limited to physical and mental consequences; rather, it also affects family and national economics [9]. Health professionals in health care institutes must manage the maintenance of plasma glucose levels among T2DM patients and blood pressure among HT patients using different regiments of drugs for their entire lives. With these demands, there are required numbers of health professionals and large amount of financial input needed to operate the treatment and care system each year. Patients need to frequently attend a clinic to meet and receive care from a doctor. Otherwise, many complications could possibly develop, resulting in intensive and complicated methods of treatment and care.

In 2014, the WHO estimated that 422 million people worldwide were suffering from T2DM, which accounted for $8.5 \%$ of the prevalence among people over 18 years old. The prevalence is increasing among people aged $>30$ years old, particularly in low- and middle-income countries. People aged $\geq 60$ years old are also commonly defined as a vulnerable population for T2DM [2]. Commonly, T2DM is a disease that progresses slowly from its onset, and it may be diagnosed several years later. T2DM is a major cause of other health problems, such as blindness, kidney failure, heart attacks, stroke, and lower limb amputation. The WHO also reported that 1.6 million deaths were directly caused by diabetes, and almost half of all deaths attributable to high blood glucose occurred before the age of 70 years [2]. This finding reflects the need to regularly investigate those vulnerable to an early diagnosis and determine ways of obtaining a better prognosis. In 2016, the total T2DM prevalence among the Thai population was $9.6 \%$ : $9.1 \%$ in males and $10.1 \%$ in females. The total number of deaths caused by T2DM was 20,570 cases; in the 30-69 year age group, the number of deaths was 8,120 cases (3,610 males, 4,510 females) and in the $\geq 70$ years age group, the number of deaths was 12,450 cases (4,760 males, 7,690 females). Moreover, total number of deaths attributable to high blood glucose was 35,640 cases; in the 30-69 year age group, the number of deaths was 13,810 cases $(7,220$ males, 6,590 females) and in the $\geq 70$ years age group, the number of deaths was 21,830 cases (9,430 males, 12,400 females) [10]. The average cost of each T2DM case in attending hospital services per year was 598US\$ for an independent case and 2,700US\$ for a disabled case. Therefore, Thailand spends a large amount of money on the health care system annually [11].

High blood pressure is a key risk factor for many diseases, including heart attack and stroke. In 2017, WHO estimated that more than one billion people had HT caused $12.8 \%$ of all deaths and accounted for 57 million disability-adjusted life years (DALYs) or a total of $3.7 \%$ DALYs every year [12]. Thailand reported that $29.0 \%$ of adult Thais had HT, and only $37.0 \%$ for those people who had been diagnosed had their blood pressure under control in 2017 [13]. The number of resistant HT patients in all health institutes in the entire country has increased from 3,946,902 cases in 2013 to 5,584,007 cases in 2017 [13]. The statistics represent the full picture of the situation in Thailand, but there is no information available on any specific subgroup of populations, such as the hill tribe population.

The hill tribe people are those who have migrated from the southern region of China to Thailand in the past century [14]. They are divided into six different main groups: Akha, Lahu, Karen, Hmong, Yao, and Lisu [15]. Approximately 2.5 million of the hill tribe people were living in Thailand in 2017 [16]. They have their own culture, language and lifestyles, particularly in daily cooking. Some tribes use a high volume of oil for cooking, whereas other tribes use a high volume of salt for their daily food [14, 17]. Most of them have similar cultural patterns in terms of using alcohol, particularly for religious rituals [18].

In 2017, the hill tribe elderly populations lived according to their own traditional lifestyle and living environment. They consumed drinks and foods prepared traditionally. Individual health care was mainly based on their local healing patterns. With the problems of distance, language and discrimination, their access to the Thai health care system was poor [19]. Therefore, access to modern medical care is not common, especially for those who live very far from the city. Ultimately, the findings of the study could support the development of the health care service system for the hill tribe elderly populations. The findings could also be used for the development of DM and HT prevention and control measures in these populations. Currently, there is no available information about T2DM and HT among these population groups. Therefore, the study aimed to estimate the prevalence and factors associated with DM and HT among the hill tribe elderly populations in northern Thailand. 


\section{Methods}

\section{Study design and participants Study design}

A cross-sectional study was conducted to gather information from the selected subjects.

\section{Study setting}

The study was conducted along 16 districts in Chiang Rai Province, which is located in Thailand.

\section{Study population}

The study population was comprised of hill tribe elderly adults aged $\geq 60$ years old who had lived in the study setting for at least 3 years.

\section{Eligible population}

Elderly adults with the following characteristics were eligible for the study: a) being classified as a member of the hill tribe by verbal confirmation, b) being $\geq 60$ years old, c) living in the study area for 3 years at the date of data collection, and d) having the ability to provide essential information. Those who had been diagnosed with type 1 diabetes mellitus, which requires daily administration of insulin, were excluded from the study.

\section{Sample size}

The sample size was calculated by Epi-Info version 7.2 (US Centers for Disease Control and Prevention, Atlanta, GA). By setting the alpha error at 0.05 , the power at 0.8 , the previous prevalence of $\mathrm{T} 2 \mathrm{DM}$ among the exposed group at $18.0 \%$, and the prevalence among the unexposed group at $0.07 \%$ [20], the sample size was calculated to be a minimum of 705 participants. Increasing the sample size by $10.0 \%$ for error resulted in 775 participants required.

Since the sample size was calculated at 775 participants, at least 130 participants were needed in each tribe.

\section{Sample selection and preparing the participants}

The list of the hill tribe villages in Chiang Rai Province was requested from the Hill Tribe Welfare and Development Center in Chiang Rai [21]. There were 749 hill tribe villages in Chiang Rai, which breakdown into 316 Lahu villages, 243 Akha villages, 63 Yao villages, 56 Hmong villages, 36 Karen villages, and 35 Lisu villages. In 2016, a total of 41,366 hill tribe families lived in Chiang Rai Province.

Permission to access the villages had been granted by the District Government Officer. Sixty hill tribe villages, or 10 villages in each tribe, were selected by a simple random method. A village headman was contacted and informed of all essential information regarding the research objective and its protocol. The list of elderly people who met the inclusion and exclusion criteria in the village was sent to the researcher. A simple random method was used to select 13 individuals in each village, after which they were invited to participate in the study. After informing the village headman about the research objectives and protocols, some tribes collected more than the minimum required sample size: Lahu (an excess of 3 participants) and Hmong (an excess of 10 participants). Those who agreed to join the project were informed of all research processes, including the preparation of NPO (nothing per oral) for at least 8 hours for the blood specimen collection on the next day (Fig. 1).

Six research assistants fluent in Thai and in one of the six hill tribal languages were recruited. Selected research assistants were trained in procedures, and the required documents were completed three days before working in the field. Most of the hill tribe elderly populations do not speak Thai. Therefore, there was a need to obtain complete information using the research assistants. Recruiting young adults to help as research assistants was possible because hill tribe community members younger than 25 years old had already completed secondary level education in Thai schools.

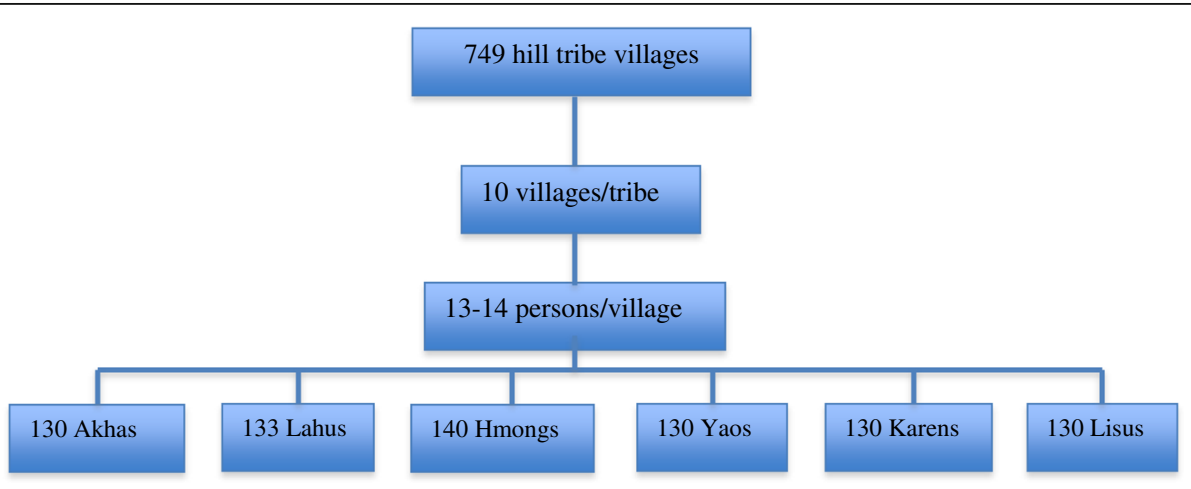

Fig. 1 Flowchart of participants' selection. Sixty hill tribe villages were randomly selected from 749 villages, and 13-14 elderly people in each village were recruited into the study 


\section{Measurement \\ Research instruments}

A questionnaire, physical examination form, a manual sphygmomanometer, and a 5-mL blood specimen served as research instruments. A questionnaire was developed from the review literature. After completion of the first draft, the validity was detected by the item-objective congruence (IOC) technique in which three external experts in relevant fields verified the validity. Questions with scores less than 0.5 were excluded, those with scores 0.5-0.69 were revised, and those with scores greater than 0.7 were defined as acceptable to use. The questionnaire was also tested for reliability by pilot testing it in 15 similar participants in the Ban San Ti Suk hill tribe village using the test-retest method. Questions with Cronbach's alpha $\geq 0.5$ were included in the form. Ultimately, there were 28 questions in three parts included in the questionnaire, which presented an overall Cronbach's alpha of 0.77 .

In the first parts, 13 questions were used to collect participants' general information, such as age, sex, education, and religion. Fifteen questions were included in the second part, including questions about health behaviors such as "Do you smoke?", "Do you drink alcohol?", and "Do you use methamphetamine?". In these questions, the three answer choices were "Yes", "Ever in the past", and "No".

Several questions were asked regarding daily food consumption and exercise, such as "Do you usually eat a salty diet?", "Do you favor having greasy food?", and "Do you like to eat sweet food?" Two answer choices were provided for the questions, "Yes" and "No". However, for exercise practices, the three answer choices were "No", "Highly active physical work, such as farmer and labor", and "Yes".

Questions on medications, history of T2DM and HT, and parental history of T2DM and HT were also included. To confirm the diagnosis, all participants who responded that they had T2DM and HT were asked to present the log-book from a hospital. In Thailand, all DM and HT cases are provided individual log-books to use to collect medical information and make appointments.

The last part consisted of twenty-one items of a physical examination form, which is used at Mae Fah Luang University Hospital. This form resembles a checklist and can include more information if required. A manual sphygmomanometer was used for assessing blood pressure.

\section{Variables and measurements}

T2DM in the study was identified by the following: a) having no history of a medical diagnosis, such as type 1 diabetes mellitus, at a particularly early age after birth, b) having shown a fasting plasma glucose $\geq 126 \mathrm{mg} / \mathrm{dL}$ twice on different days [20].
Blood pressure was assessed twice in all participants with a 15-minute gap between assessments, and in case systolic and/or diastolic blood pressures were greater than $90 \mathrm{mmHg}$ and/or $150 \mathrm{mmHg}$, respectively, it was assessed again after 15 minutes of rest following the $2^{\text {nd }}$ assessment. Participants with $90 \mathrm{mmHg}$ and/or 140 $\mathrm{mmHg}$ of systolic and diastolic blood pressures, respectively, were diagnosed as HT patients [22].

Body mass index (BMI) was classified into three categories, according to the WHO guidelines for Asian populations: underweight $(\mathrm{BMI} \leq 18.5)$, normal weight (BMI=18.51-22.99), and overweight (BMI 2 23.00) [23].

A 5-mL blood specimen was collected from a peripheral vein puncture. After blood was drawn, a 3-mL blood specimen was collected and stored in a sodium fluoride tube to detect fasting plasma glucose. Another 2-mL blood specimen was collected and stored in a clot blood clot tube for detecting lipid profiles. Uric acid, cholesterol, and triglycerides were assessed in $\mathrm{mg} / \mathrm{dL}$. Participants with uric acid $\geq 7 \mathrm{mg} / \mathrm{dL}$, cholesterol $\geq 200 \mathrm{mg} / \mathrm{dL}$, and triglycerides $\geq 200 \mathrm{mg} / \mathrm{dL}$ were defined as a high-level group [24]. Participants with fasting plasma glucose $\geq 126 \mathrm{mg} / \mathrm{dL}$ were asked to provide another blood specimen within a week to determine type 2 diabetes stage.

\section{Procedures}

\section{Data gathering procedures}

After the consent form was obtained, a 5-mL blood specimen was collected. Participants were asked to complete the questionnaire in a private room in the village with the help of the research assistants. A trained physician examined the physical health of all participants in a proper room. A small gift was given to participants after they completed the questionnaire.

\section{Statistical analysis}

Descriptive statistics, such as the means, minimums, maximums, standard deviations, and percentages, were used to explain the general characteristics of the participants. Chi-square tests and logistic regressions were used to detect the associations between variables at the significance level $\alpha=0.05$. Logistic regression was used to detect the associations between variables in both univariate and multivariate models. The "ENTER" mode was used to select the significant variables in the model. The significance level (alpha) was set at 0.05 in both univariate and multivariate analyses. Variables that were found to be significant in the univariate analysis were retained in the multivariate analysis. In the multivariate model, the most nonsignificant variable was deleted from the model before running the second step. The model was analyzed until all remaining variables were found to be significant at an alpha level of 0.05 , and the results were interpreted. 
Table 1 General characteristics of the study participants

\begin{tabular}{|c|c|c|}
\hline Characteristics & Number & Percent \\
\hline Total & 793 & 100.0 \\
\hline \multicolumn{3}{|l|}{ Sex } \\
\hline Male & 393 & 49.6 \\
\hline Female & 400 & 50.4 \\
\hline \multicolumn{3}{|l|}{ Thai ID card } \\
\hline Yes & 745 & 93.9 \\
\hline No & 48 & 6.1 \\
\hline \multicolumn{3}{|l|}{ Tribe } \\
\hline Akha & 130 & 16.4 \\
\hline Lahu & 133 & 16.8 \\
\hline Hmong & 140 & 17.6 \\
\hline Yao & 130 & 16.4 \\
\hline Karen & 130 & 16.4 \\
\hline Lisu & 130 & 16.4 \\
\hline \multicolumn{3}{|l|}{ Age (years) } \\
\hline $60-69$ & 410 & 51.7 \\
\hline $70-79$ & 279 & 35.2 \\
\hline$\geq 80$ & 104 & 13.1 \\
\hline \multicolumn{3}{|l|}{ Religion } \\
\hline Buddhism & 567 & 71.5 \\
\hline Christianity & 225 & 28.4 \\
\hline Islam & 1 & 0.1 \\
\hline \multicolumn{3}{|l|}{ Education } \\
\hline None & 739 & 93.8 \\
\hline Primary School & 41 & 5.2 \\
\hline High School & 8 & 1.0 \\
\hline \multicolumn{3}{|l|}{ Resides with } \\
\hline Child & 559 & 70.5 \\
\hline Cousin & 12 & 1.5 \\
\hline Spouse & 174 & 21.9 \\
\hline Alone & 48 & 6.1 \\
\hline \multicolumn{3}{|l|}{ Marital status } \\
\hline Single & 15 & 1.9 \\
\hline Married & 524 & 66.8 \\
\hline Divorced & 20 & 2.5 \\
\hline Widow & 226 & 28.8 \\
\hline \multicolumn{3}{|c|}{ Number of family member (persons) } \\
\hline 1 & 40 & 5.0 \\
\hline 2 & 116 & 14.6 \\
\hline $3-5$ & 301 & 38.0 \\
\hline 6 & 336 & 42.4 \\
\hline \multicolumn{3}{|l|}{ Occupation } \\
\hline Unemployed (retired) & 499 & 62.9 \\
\hline Farmer & 252 & 31.8 \\
\hline
\end{tabular}

Table 1 General characteristics of the study participants (Continued)

\begin{tabular}{lll}
\hline Characteristics & Number & Percent \\
\hline Merchant & 11 & 1.4 \\
Labor & 19 & 2.4 \\
Other & 12 & 1.5 \\
Monthly family income (baht) & & \\
0 & 69 & 8.7 \\
$\leq 5,000$ & 707 & 89.2 \\
$\geq 5,001$ & 17 & 2.1 \\
Debt (baht) & & \\
0 & 673 & 84.9 \\
$\leq 5,000$ & 14 & 1.8 \\
$5,001-10,000$ & 11 & 1.4 \\
$10,001-50,000$ & 58 & 7.3 \\
$\geq 50,001$ & 37 & 4.6 \\
\hline
\end{tabular}

\section{Results}

Characteristics of participants

In total, 793 participants were recruited into the study. Proportions of participants were mostly equal by sex and among the six tribes. A few people had no Thai identification card (6.1\%), with an equal proportion among the tribes. The majority were aged $60-69$ years $(51.7 \%)$, with an average age of 70.1 years (range $=60-100, \mathrm{SD}=7.57$, $\max =100$, and $\min =60)$. The majority of the sample practiced Buddhism (71.5\%) and had no education (94.8\%). A few people lived alone (6.1\%), and most participants were married (66.8\%). Regarding economic status, $89.2 \%$ had an income of $\leq 5,000$ baht/month (mean=1,129 baht, $\mathrm{SD}=1,273$ ), and $84.9 \%$ had no debt (Table 1 ).

There were no statistical differences in the distribution of participants according to sex and tribe in three different age categories (60-69, 70-79, and $\geq 80$ years). A few of the hill tribe elderly adults had the ability to communicate in Thai: $19.5 \%$ could speak, $19.5 \%$ could understand, $2.0 \%$ could read, and $1.6 \%$ could write fluently. Males had significantly better Thai communication skills than females in all four domains: speaking, understanding, reading, and writing.

The prevalence of T2DM and HT was $16.8 \%$ and $45.5 \%$, respectively. Seventy-five participants had been diagnosed with T2DM before being recruited into the study. Among these participants, 8 (10.6\%) had high fasting glucose or were unable to control blood glucose after medication. Fifty-five participants (7.7\%) were detected as new T2DM cases (Table 2). However, 18 participants (1.2\%) could not draw blood specimens.

Two hundred and forty participants (30.3\%) had been diagnosed with $\mathrm{HT}$, among whom $37.9 \%$ were unable to control their blood pressure after medication. After 
Table 2 Prevalence of T2DM and HT among the participants

\begin{tabular}{lll}
\hline Chracteristics & Number & Percent \\
\hline Medical history of T2DM & & \\
No & 718 & 90.5 \\
Yes & 75 & 9.5
\end{tabular}

Effective control of blood glucose by daily medication

\begin{tabular}{lcc} 
No & 8 & 10.6 \\
Yes & 67 & 89.4 \\
asting plasma glucose level among non-DM diagnosed & \\
Normal & 645 & 89.8 \\
High (T2DM) & 55 & 7.7 \\
(Missing=18, 2.5\%) & \\
Prevalence of T2DM=16.8\% & \\
Medical history of HT & \\
No & 553 & 69.7 \\
$\quad$ Yes & 240 & 30.3 \\
Effective control of blood pressure by daily medication & \\
$\quad$ No & 91 & 37.9 \\
Yes & 149 & 62.1 \\
Blood pressure level among non-HT diagnosed & \\
Normal & 432 & 78.1 \\
High (HT) & 121 & 21.9 \\
Prevalence of HT=45.5\% & & \\
Having both T2DM and HT & 70 & 9.0 \\
\hline
\end{tabular}

a The overall prevalence of T2DM among the participants

${ }^{b}$ The overall prevalence of $\mathrm{HT}$ among the participants

those who had no history of HT diagnosis and medication were seen, 121 participants $(21.9 \%)$ were detected as new HT cases. Finally, 70 cases $(9.0 \%)$ were determined to have both T2DM and HT: 36 males and 34 females (Table 2).

There was statistical significance in the proportion of participants with T2DM and HT by sex and tribe. Only the participants with T2DM showed a statistically significant difference in proportion (Table 3).

Health behaviors among the participants indicated that $19.7 \%$ smoked, $14.6 \%$ drank alcohol, $44.9 \%$ ate uncooked food, $23.8 \%$ chewed tobacco, and $10.1 \%$ did not exercise regularly. A comparison of health behaviors such as smoking, alcohol use, eating uncooked food, and regular exercise among the tribes showed statistically significant differences (Table 4). Additionally, there were significant sex differences in the following health behaviors: smoking; alcohol use; the consumption of uncooked food, salty food, greasy food, and sweet food; opium use; chewing tobacco; and regular exercise (Table 5).

Most participants had moderate levels of health-related knowledge, attitudes, and practices. Only the distribution of attitudes by tribe showed statistical significance (Table 6).
Table 3 Comparison of T2DM and HT by participants' characteristics

\begin{tabular}{|c|c|c|c|c|c|c|c|c|}
\hline \multirow[t]{2}{*}{ Characteristic } & \multicolumn{2}{|l|}{ T2DM } & \multirow[t]{2}{*}{$x^{2}$} & \multirow[t]{2}{*}{$p$-value } & \multicolumn{2}{|l|}{ HT } & \multirow[t]{2}{*}{$x^{2}$} & \multirow[t]{2}{*}{$p$-value } \\
\hline & $\begin{array}{l}\text { Yes } \\
(\%)\end{array}$ & $\begin{array}{l}\text { No } \\
(\%)\end{array}$ & & & $\begin{array}{l}\text { Yes } \\
(\%)\end{array}$ & $\begin{array}{l}\text { No } \\
(\%)\end{array}$ & & \\
\hline \multicolumn{9}{|l|}{ Sex } \\
\hline Male & $\begin{array}{l}66 \\
(17.3)\end{array}$ & $\begin{array}{l}316 \\
(82.7)\end{array}$ & 0.13 & 0.712 & $\begin{array}{l}164 \\
(41.7)\end{array}$ & $\begin{array}{l}229 \\
(58.3)\end{array}$ & 4.52 & $0.034^{*}$ \\
\hline Female & $\begin{array}{l}64 \\
(16.3)\end{array}$ & $\begin{array}{l}329 \\
(83.7)\end{array}$ & & & $\begin{array}{l}197 \\
(49.3)\end{array}$ & $\begin{array}{l}203 \\
(50.7)\end{array}$ & & \\
\hline \multicolumn{9}{|l|}{ Age (years) } \\
\hline $60-69$ & $\begin{array}{l}75 \\
(18.8)\end{array}$ & $\begin{array}{l}324 \\
(81.2)\end{array}$ & 2.49 & 0.287 & $\begin{array}{l}173 \\
(42.2)\end{array}$ & $\begin{array}{l}237 \\
(57.8)\end{array}$ & 4.25 & 0.119 \\
\hline $70-79$ & $\begin{array}{l}39 \\
(14.3)\end{array}$ & $\begin{array}{l}234 \\
(85.7)\end{array}$ & & & $\begin{array}{l}134 \\
(48.0)\end{array}$ & $\begin{array}{l}145 \\
(52.0)\end{array}$ & & \\
\hline$\geq 80$ & $\begin{array}{l}16 \\
(15.5)\end{array}$ & $\begin{array}{l}87 \\
(84.5)\end{array}$ & & & $\begin{array}{l}54 \\
(51.9)\end{array}$ & $\begin{array}{l}50 \\
(48.1)\end{array}$ & & \\
\hline \multicolumn{9}{|l|}{ Tribe } \\
\hline Akha & $\begin{array}{l}11 \\
(8.6)\end{array}$ & $\begin{array}{l}117 \\
(91.4)\end{array}$ & 24.48 & $<0.001^{*}$ & $\begin{array}{l}61 \\
(46.9)\end{array}$ & $\begin{array}{l}69 \\
(53.1)\end{array}$ & 26.45 & $<0.001^{*}$ \\
\hline Lahu & $\begin{array}{l}26 \\
(19.5)\end{array}$ & $\begin{array}{l}107 \\
(80.5)\end{array}$ & & & $\begin{array}{l}61 \\
(45.9)\end{array}$ & $\begin{array}{l}72 \\
(54.1)\end{array}$ & & \\
\hline Hmong & $\begin{array}{l}11 \\
(8.1)\end{array}$ & $\begin{array}{l}124 \\
(91.9)\end{array}$ & & & $\begin{array}{l}42 \\
(30.0)\end{array}$ & $\begin{array}{l}98 \\
(70.0)\end{array}$ & & \\
\hline Yao & $\begin{array}{l}26 \\
(21.5)\end{array}$ & $\begin{array}{l}95 \\
(78.5)\end{array}$ & & & $\begin{array}{l}74 \\
(56.9)\end{array}$ & $\begin{array}{l}56 \\
(43.1)\end{array}$ & & \\
\hline Karen & $\begin{array}{l}34 \\
(26.4)\end{array}$ & $\begin{array}{l}95 \\
(73.6)\end{array}$ & & & $\begin{array}{l}52 \\
(40.0)\end{array}$ & $\begin{array}{l}78 \\
(60.0)\end{array}$ & & \\
\hline Lisu & $\begin{array}{l}22 \\
(17.1)\end{array}$ & $\begin{array}{l}107 \\
(82.9)\end{array}$ & & & $\begin{array}{l}71 \\
(54.6)\end{array}$ & $\begin{array}{l}59 \\
(45.4)\end{array}$ & & \\
\hline
\end{tabular}

*Significance level at $a=0.05$

With regard to the physical health and medical history among the participants, $45.0 \%$ were overweight, $6.8 \%$ were disabled persons, $15.0 \%$ had sleeping problems, $9.7 \%$ had cataracts, $28.7 \%$ had hearing problems, and $43.3 \%$ had tooth problems (Table 7).

There were statistically significant differences in the quality of uric acid and cholesterol according to sex, age category, and tribe. A greater proportion of males, individuals in higher age categories, and Lahu and Lisu tribe members had high uric acid levels than did females, those in younger age categories, and members of other tribes. Only age category and tribe showed significant differences on the level of triglycerides; a greater proportion of those in lower age categories had high cholesterol than those in higher age categories. A greater proportion of members of the Lahu and Akha tribes were in the high cholesterol group compared to those in the remaining tribes (Table 8).

In the multivariate model, five factors were associated with T2DM: tribe, exercise, BMI, parental history of T2DM, and triglycerides. The Lahu, Yao, Karen, and Lisu tribes had greater odds of developing T2DM than the Akha tribe, with $\quad \mathrm{OR}_{\mathrm{adj}}=2.89 \quad(95 \% \mathrm{CI}=1.32-6.33)$, $\mathrm{OR}_{\mathrm{adj}}=3.47 \quad(95 \% \mathrm{CI}=1.58-7.62), \quad \mathrm{OR}_{\mathrm{adj}}=5.03 \quad(95 \% \mathrm{CI}=$ 
Table $\mathbf{4}$ Characteristics of health behaviors by tribe

\begin{tabular}{|c|c|c|c|c|c|c|c|c|c|c|c|c|c|c|c|c|}
\hline \multirow[t]{3}{*}{ Health behaviors } & \multicolumn{14}{|c|}{ Tribe } & \multirow[t]{3}{*}{$x^{2}$} & \multirow[t]{3}{*}{$p$-value } \\
\hline & \multicolumn{2}{|c|}{ Total } & \multicolumn{2}{|c|}{ Akha } & \multicolumn{2}{|c|}{ Lahu } & \multicolumn{2}{|c|}{ Hmong } & \multicolumn{2}{|l|}{ Yao } & \multicolumn{2}{|c|}{ Karen } & \multicolumn{2}{|l|}{$\underline{\text { Lisu }}$} & & \\
\hline & $\mathrm{n}$ & $\%$ & $\mathrm{n}$ & $\%$ & $\mathrm{n}$ & $\%$ & $\mathrm{n}$ & $\%$ & $n$ & $\%$ & $n$ & $\%$ & $n$ & $\%$ & & \\
\hline \multicolumn{17}{|l|}{ Smoking } \\
\hline No & 486 & 61.3 & 94 & 19.3 & 70 & 14.4 & 106 & 21.8 & 72 & 14.8 & 48 & 9.9 & 96 & 19.8 & 79.02 & $<0.001^{*}$ \\
\hline Ever in the past & 151 & 19.0 & 12 & 7.9 & 33 & 21.9 & 11 & 7.3 & 29 & 19.2 & 50 & 33.1 & 16 & 10.6 & & \\
\hline Yes & 156 & 19.7 & 24 & 15.4 & 30 & 19.2 & 23 & 14.7 & 29 & 18.6 & 32 & 20.5 & 18 & 11.5 & & \\
\hline \multicolumn{17}{|l|}{ Alcohol use } \\
\hline No & 538 & 67.8 & 99 & 18.4 & 92 & 17.1 & 109 & 20.3 & 88 & 16.4 & 77 & 14.3 & 73 & 13.6 & 43.93 & $<0.001^{*}$ \\
\hline Ever & 139 & 17.5 & 13 & 9.4 & 29 & 20.9 & 14 & 10.1 & 17 & 12.2 & 25 & 18.0 & 41 & 29.5 & & \\
\hline Yes & 116 & 14.6 & 18 & 15.5 & 12 & 10.3 & 17 & 14.7 & 25 & 21.6 & 28 & 24.1 & 16 & 13.8 & & \\
\hline \multicolumn{17}{|l|}{ Methamphetamine use } \\
\hline No & 776 & 97.9 & 124 & 16.0 & 132 & 17.0 & 137 & 17.7 & 126 & 16.4 & 128 & 16.5 & 129 & 16.6 & 12.15 & 0.275 \\
\hline Ever in the past & 2 & 0.3 & 0 & 0.0 & 0 & 0.0 & 0 & 0.0 & 1 & 50.0 & 1 & 50.0 & 0.0 & 0.0 & & \\
\hline Yes & 15 & 1.9 & 6 & 40.0 & 1 & 6.7 & 3 & 20.0 & 3 & 20.0 & 1 & 6.7 & 1 & 6.7 & & \\
\hline \multicolumn{17}{|l|}{ Opium use } \\
\hline No & 723 & 91.2 & 112 & 15.5 & 125 & 17.3 & 124 & 17.2 & 115 & 15.9 & 123 & 17.0 & 124 & 17.2 & 15.77 & 0.106 \\
\hline Ever in the past & 54 & 6.8 & 12 & 22.2 & 6 & 11.1 & 12 & 22.2 & 12 & 22.2 & 7 & 13.0 & 5 & 9.3 & & \\
\hline Yes & 16 & 2.0 & 6 & 37.5 & 2 & 12.5 & 4 & 25.0 & 3 & 18.8 & 0 & 0.0 & 1 & 6.3 & & \\
\hline \multicolumn{17}{|l|}{ Eating uncooked food } \\
\hline No & 385 & 48.5 & 79 & 20.5 & 74 & 19.2 & 68 & 17.7 & 69 & 17.9 & 43 & 11.2 & 52 & 13.5 & 29.65 & $<0.001^{*}$ \\
\hline Ever in the past & 52 & 6.6 & 5 & 9.6 & 6 & 11.5 & 9 & 17.3 & 8 & 15.4 & 11 & 21.2 & 13 & 25.0 & & \\
\hline Yes & 356 & 44.9 & 46 & 12.9 & 53 & 14.9 & 63 & 17.7 & 53 & 14.9 & 76 & 21.3 & 65 & 18.3 & & \\
\hline \multicolumn{17}{|l|}{ Chewing } \\
\hline No & 604 & 76.2 & 70 & 11.6 & 108 & 17.9 & 135 & 22.4 & 128 & 21.2 & 100 & 16.6 & 63 & 10.4 & 159.80 & $<0.001^{*}$ \\
\hline Yes & 189 & 23.8 & 60 & 31.7 & 25 & 13.2 & 5 & 2.6 & 2 & 1.1 & 30 & 15.9 & 67 & 35.4 & & \\
\hline \multicolumn{17}{|l|}{ Regular exercise } \\
\hline No & 80 & 10.1 & 22 & 27.5 & 7 & 8.8 & 15 & 18.8 & 7 & 8.8 & 22 & 27.5 & 7 & 8.8 & 37.50 & $<0.001^{*}$ \\
\hline Yes & 433 & 54.6 & 68 & 15.7 & 88 & 20.3 & 75 & 17.3 & 66 & 15.2 & 54 & 12.5 & 82 & 18.9 & & \\
\hline Highly active physical work & 280 & 35.3 & 40 & 14.3 & 38 & 13.6 & 50 & 17.9 & 57 & 20.4 & 54 & 19.3 & 41 & 14.6 & & \\
\hline
\end{tabular}

*Significance level at $\mathrm{a}=0.05$

$2.35-10.78)$, and $\mathrm{OR}_{\mathrm{adj}}=2.73(95 \% \mathrm{CI}=1.22-6.07)$ respectively. Those who were overweight had greater odds of developing T2DM than those with normal weight, with $\mathrm{OR}_{\mathrm{adj}}=2.08$ (95\%CI=1.32-3.27). Those who had a parental history of T2DM had greater odds of developing T2DM than those who did not, with $\mathrm{OR}_{\mathrm{adj}}=1.55$ (95\%CI=1.17-2.10). Those with high cholesterol had greater odds of developing T2DM than those with low cholesterol, with $\mathrm{OR}_{\mathrm{adj}}=1.73$ $(95 \% \mathrm{CI}=1.10-2.73)$. Those who engaged in high levels of physical activity and exercise had lower odds of developing T2DM than those who did not, with $\mathrm{OR}_{\mathrm{adj}}=0.48$ $(95 \% \mathrm{CI}=0.25-0.91)$ and $\mathrm{OR}_{\mathrm{adj}}=0.45(95 \% \mathrm{CI}=0.24-0.83)$, respectively (Table 9).

Four factors were found to be associated with HT after controlling for all possible confounding variables: sex, dietary salt intake, BMI, and parental history of HT. Females had greater odds of developing HT than males, with $\mathrm{OR}_{\mathrm{adj}}=1.29 \quad(95 \% \mathrm{CI}=1.01-1.68)$. Those who had dietary salt intake had greater odds of developing HT than those who did not, with $\mathrm{OR}_{\mathrm{adj}}=1.48$ (95\%CI=1.14-2.00). Those who were overweight had greater odds of developing HT than those with normal weight, with $\mathrm{OR}_{\mathrm{adj}}=1.37(95 \% \mathrm{CI}=1.01-1.90)$, and those who had a parental history of HT had greater odds of developing HT than those who did not, with $\mathrm{OR}_{\mathrm{adj}}=3.38$ $(95 \% \mathrm{CI}=2.81-4.48)$ (Table 10).

\section{Discussion}

Members of the hill tribe elderly population are living with a high burden of T2DM and HT in Thailand. There 
Table 5 Comparison of health behavior by sex

\begin{tabular}{|c|c|c|c|c|c|c|c|c|}
\hline \multirow[t]{2}{*}{ Health behvaior } & \multicolumn{2}{|c|}{ Total } & \multicolumn{2}{|c|}{ Male } & \multicolumn{2}{|c|}{ Female } & \multirow[t]{2}{*}{$x^{2}$} & \multirow[t]{2}{*}{$p$-value } \\
\hline & $n$ & $\%$ & $n$ & $\%$ & $n$ & $\%$ & & \\
\hline \multicolumn{9}{|l|}{ Smoking } \\
\hline No & 486 & 61.3 & 151 & 31.1 & 335 & 68.9 & \multirow[t]{3}{*}{173.52} & \multirow[t]{3}{*}{$<0.001^{*}$} \\
\hline Ever in the past & 151 & 19.0 & 125 & 82.8 & 26 & 17.2 & & \\
\hline Yes & 156 & 19.7 & 117 & 75.0 & 39 & 25.0 & & \\
\hline \multicolumn{9}{|l|}{ Alcohol use } \\
\hline No & 538 & 67.8 & 169 & 31.4 & 369 & 68.6 & \multirow[t]{3}{*}{222.02} & \multirow[t]{3}{*}{$<0.001^{*}$} \\
\hline Ever in the past & 139 & 17.5 & 117 & 84.2 & 22 & 15.8 & & \\
\hline Yes & 116 & 14.6 & 107 & 92.2 & 9 & 7.8 & & \\
\hline \multicolumn{9}{|l|}{ Consumption of uncooked food } \\
\hline No & 385 & 48.5 & 106 & 27.5 & 279 & 72.5 & \multirow[t]{3}{*}{145.24} & \multirow[t]{3}{*}{$<0.001^{*}$} \\
\hline Ever in the past & 52 & 6.6 & 37 & 71.2 & 15 & 28.8 & & \\
\hline Yes & 356 & 44.9 & 250 & 70.2 & 106 & 29.8 & & \\
\hline \multicolumn{9}{|l|}{ Salty food } \\
\hline No & 282 & 35.6 & 106 & 37.6 & 176 & 62.4 & \multirow[t]{2}{*}{25.05} & \multirow[t]{2}{*}{$<0.001^{*}$} \\
\hline Yes & 511 & 64.4 & 287 & 56.2 & 224 & 43.8 & & \\
\hline \multicolumn{9}{|l|}{ Greasy food } \\
\hline No & 297 & 37.5 & 194 & 65.3 & 103 & 34.7 & \multirow[t]{2}{*}{47.12} & \multirow[t]{2}{*}{$<0.001^{*}$} \\
\hline Yes & 496 & 62.5 & 199 & 40.1 & 297 & 59.9 & & \\
\hline \multicolumn{9}{|l|}{ Sweet food } \\
\hline No & 391 & 49.3 & 216 & 55.2 & 175 & 44.8 & \multirow[t]{2}{*}{9.96} & \multirow[t]{2}{*}{$0.0016^{*}$} \\
\hline Yes & 402 & 50.7 & 177 & 44.0 & 225 & 56.0 & & \\
\hline \multicolumn{9}{|l|}{ Opium use } \\
\hline No & 723 & 91.2 & 339 & 46.9 & 384 & 53.1 & \multirow[t]{3}{*}{23.95} & $<0.001^{*}$ \\
\hline Ever in the past & 54 & 6.8 & 43 & 79.6 & 11 & 20.4 & & \\
\hline Yes & 16 & 2.0 & 11 & 68.8 & 5 & 31.3 & & \\
\hline Methamphetamine use & & & & & & & & \\
\hline No & 776 & 97.9 & 381 & 49.1 & 395 & 50.9 & 3.69 & 0.079 \\
\hline Yes & 17 & 2.1 & 12 & 70.6 & 5 & 29.4 & & \\
\hline Chewing & & & & & & & & \\
\hline No & 604 & 76.2 & 313 & 51.8 & 291 & 48.2 & 5.19 & $0.023^{*}$ \\
\hline Yes & 189 & 23.8 & 80 & 42.3 & 109 & 57.7 & & \\
\hline Regular exercise & & & & & & & & \\
\hline No & 433 & 54.6 & 184 & 42.5 & 249 & 57.5 & 26.05 & $<0.001^{*}$ \\
\hline Highly active physical work & 280 & 35.3 & 173 & 61.8 & 107 & 38.2 & & \\
\hline Yes & 80 & 10.1 & 36 & 45.0 & 44 & 55.0 & & \\
\hline
\end{tabular}

*Significance level at $\alpha=0.05$

are several factors associated with HT and T2DM, such as behaviors related to daily living, culture and food practices. Most members of the hill tribe elderly population have no education and low economic status. Very few have Thai ID cards, which is usually used to access all public services in Thailand, including health care services [17]. Only one-fourth of the participants were able to speak and understand Thai, and a few people could read and write in Thai. The prevalence of T2DM and HT was $16.8 \%$ and $45.5 \%$, respectively, of which $7.7 \%$ and $21.9 \%$ represented the incident rates for T2DM and HT, respectively. Moreover, 9.3\% of T2DM participants and $37.9 \%$ of HT participants could not control their plasma glucose and blood pressure after having daily medication. The comorbidity rate was approximately one-fourth of the participants who used alcohol and 
Table 6 Comparison on knowledge, attitudes, and practices regarding health among tribes

\begin{tabular}{|c|c|c|c|c|c|c|c|c|c|c|c|c|c|c|c|c|}
\hline \multirow[t]{3}{*}{ KAP } & & & \multicolumn{12}{|c|}{ Tribe } & \multirow[t]{3}{*}{$x^{2}$} & \multirow[t]{3}{*}{$p$-value } \\
\hline & \multicolumn{2}{|c|}{ Total } & \multicolumn{2}{|c|}{ Akha } & \multicolumn{2}{|c|}{ Lahu } & \multicolumn{2}{|c|}{ Hmong } & \multicolumn{2}{|c|}{ Yao } & \multicolumn{2}{|c|}{ Karen } & \multicolumn{2}{|c|}{ Lisu } & & \\
\hline & $n$ & $\%$ & $n$ & $\%$ & $n$ & $\%$ & $n$ & $\%$ & $n$ & $\%$ & $n$ & $\%$ & $n$ & $\%$ & & \\
\hline Total & 377 & 100.0 & 60 & 15.9 & 76 & 20.2 & 46 & 12.2 & 70 & 18.6 & 73 & 19.4 & 52 & 13.8 & & \\
\hline \multicolumn{17}{|l|}{ Knowledge } \\
\hline Low & 61 & 16.2 & 15 & 24.6 & 13 & 21.3 & 10 & 16.4 & 8 & 13.1 & 5 & 8.2 & 10 & 16.4 & 15.07 & 0.129 \\
\hline Moderate & 167 & 44.3 & 24 & 14.4 & 33 & 19.8 & 21 & 12.6 & 38 & 22.8 & 31 & 18.6 & 20 & 12.0 & & \\
\hline High & 149 & 39.5 & 21 & 14.1 & 30 & 20.1 & 15 & 10.1 & 24 & 16.1 & 37 & 24.8 & 22 & 14.8 & & \\
\hline \multicolumn{17}{|l|}{ Attitude } \\
\hline Low & 53 & 14.1 & 12 & 22.6 & 5 & 9.4 & 14 & 26.4 & 14 & 26.4 & 4 & 7.5 & 5 & 9.4 & 38.04 & $<0.001^{*}$ \\
\hline Moderate & 250 & 66.3 & 44 & 17.6 & 55 & 22.0 & 25 & 10.0 & 42 & 16.8 & 44 & 17.6 & 40 & 16.0 & & \\
\hline High & 74 & 19.6 & 4 & 5.4 & 16 & 21.6 & 7 & 9.5 & 14 & 18.9 & 25 & 33.8 & 8 & 10.8 & & \\
\hline \multicolumn{17}{|l|}{ Practice } \\
\hline Low & 47 & 12.5 & 3 & 6.4 & 8 & 17.0 & 10 & 21.3 & 9 & 19.1 & 8 & 17.0 & 9 & 19.1 & 10.51 & 0.397 \\
\hline Moderate & 267 & 70.8 & 44 & 16.5 & 56 & 21.0 & 27 & 10.1 & 49 & 18.4 & 54 & 20.2 & 37 & 13.9 & & \\
\hline High & 63 & 16.7 & 13 & 20.6 & 12 & 19.0 & 9 & 14.3 & 12 & 19.0 & 11 & 17.5 & 6 & 9.5 & & \\
\hline
\end{tabular}

*Significance level at $\mathrm{a}=0.05$

Table 7 Physical examination and medical history

\begin{tabular}{|c|c|c|c|c|c|c|c|c|}
\hline \multirow[t]{2}{*}{ Item } & \multicolumn{2}{|c|}{ Total } & \multicolumn{2}{|c|}{ Male } & \multicolumn{2}{|c|}{ Female } & \multirow[t]{2}{*}{$x^{2}$} & \multirow[t]{2}{*}{$p$-value } \\
\hline & $n$ & $\%$ & $n$ & $\%$ & $n$ & $\%$ & & \\
\hline \multicolumn{9}{|l|}{ BMI } \\
\hline Underweight & 116 & 14.6 & 62 & 53.4 & 54 & 46.6 & 3.98 & 0.137 \\
\hline Normal & 320 & 40.4 & 168 & 52.5 & 152 & 47.5 & & \\
\hline Overweight & 357 & 45.0 & 163 & 45.7 & 194 & 54.3 & & \\
\hline \multicolumn{9}{|l|}{ Disabled } \\
\hline No & 739 & 93.2 & 362 & 49.0 & 377 & 51.0 & 1.42 & 0.232 \\
\hline Yes & 54 & 6.8 & 31 & 57.4 & 23 & 42.6 & & \\
\hline \multicolumn{9}{|l|}{ Heart disease } \\
\hline No & 724 & 96.1 & 337 & 46.5 & 387 & 53.5 & 0.37 & 0.538 \\
\hline Yes & 29 & 3.9 & 16 & 55.2 & 13 & 44.8 & & \\
\hline \multicolumn{9}{|l|}{ History of TB diagnosis } \\
\hline No & 757 & 95.5 & 369 & 48.7 & 388 & 51.3 & 4.41 & $0.036^{*}$ \\
\hline Yes & 36 & 4.5 & 24 & 66.7 & 12 & 33.3 & & \\
\hline \multicolumn{9}{|l|}{ Sleeping problem } \\
\hline No & 674 & 85.0 & 356 & 52.8 & 318 & 47.2 & 19.09 & $<0.001^{*}$ \\
\hline Yes & 119 & 15.0 & 37 & 31.1 & 82 & 68.9 & & \\
\hline \multicolumn{9}{|l|}{ Eye } \\
\hline Normal & 663 & 83.6 & 328 & 49.5 & 335 & 50.5 & 0.99 & 0.804 \\
\hline Cataract & 77 & 9.7 & 36 & 46.8 & 41 & 53.2 & & \\
\hline Pterygium & 50 & 6.3 & 27 & 54.0 & 23 & 46.0 & & \\
\hline History of glaucoma & 3 & 0.4 & 2 & 66.7 & 1 & 33.3 & & \\
\hline
\end{tabular}


Table 7 Physical examination and medical history (Continued)

\begin{tabular}{|c|c|c|c|c|c|c|c|c|}
\hline \multirow[t]{2}{*}{ Item } & \multicolumn{2}{|c|}{ Total } & \multicolumn{2}{|c|}{ Male } & \multicolumn{2}{|c|}{ Female } & \multirow[t]{2}{*}{$x^{2}$} & \multirow[t]{2}{*}{$p$-value } \\
\hline & $n$ & $\%$ & $n$ & $\%$ & $n$ & $\%$ & & \\
\hline \multicolumn{9}{|c|}{ Tooth problem } \\
\hline No & 450 & 56.7 & 234 & 52.0 & 216 & 48.0 & \multirow[t]{2}{*}{2.48} & \multirow[t]{2}{*}{0.115} \\
\hline Yes & 343 & 43.3 & 159 & 46.4 & 184 & 53.6 & & \\
\hline \multicolumn{9}{|c|}{ Headache } \\
\hline No & 557 & 72.1 & 302 & 54.2 & 275 & 49.4 & \multirow[t]{2}{*}{6.55} & \multirow[t]{2}{*}{$0.010^{*}$} \\
\hline Yes & 216 & 27.9 & 91 & 42.1 & 125 & 57.9 & & \\
\hline \multicolumn{9}{|c|}{ Dizziness } \\
\hline No & 556 & 70.1 & 294 & 52.9 & 262 & 47.1 & \multirow[t]{2}{*}{8.19} & \multirow[t]{2}{*}{$0.004^{*}$} \\
\hline Yes & 237 & 29.9 & 99 & 41.8 & 138 & 58.2 & & \\
\hline \multicolumn{9}{|c|}{ Peptic ulcer } \\
\hline No & 527 & 66.5 & 278 & 52.8 & 249 & 47.2 & \multirow[t]{2}{*}{6.40} & \multirow[t]{2}{*}{$0.011^{*}$} \\
\hline Yes & 266 & 33.5 & 115 & 43.2 & 151 & 56.8 & & \\
\hline \multicolumn{9}{|c|}{ Anorexia } \\
\hline No & 707 & 89.2 & 371 & 52.5 & 336 & 47.5 & \multirow[t]{2}{*}{22.18} & \multirow[t]{2}{*}{$<0.001^{*}$} \\
\hline Yes & 86 & 10.8 & 22 & 25.6 & 64 & 74.4 & & \\
\hline \multicolumn{9}{|c|}{ History of injury } \\
\hline No & 713 & 89.9 & 349 & 48.9 & 364 & 51.1 & \multirow[t]{2}{*}{1.05} & \multirow[t]{2}{*}{0.305} \\
\hline Yes & 80 & 10.1 & 44 & 55.0 & 36 & 45.0 & & \\
\hline \multicolumn{9}{|c|}{ History of hospital admission } \\
\hline No & 310 & 39.1 & 143 & 46.1 & 167 & 53.9 & \multirow[t]{2}{*}{2.39} & \multirow[t]{2}{*}{0.122} \\
\hline Yes & 483 & 60.9 & 250 & 51.8 & 233 & 48.2 & & \\
\hline \multicolumn{9}{|c|}{ Parental history of DM } \\
\hline No & 515 & 64.9 & 262 & 50.9 & 253 & 49.1 & \multirow[t]{2}{*}{1.01} & \multirow[t]{2}{*}{0.313} \\
\hline Yes & 278 & 35.1 & 131 & 47.1 & 147 & 52.9 & & \\
\hline Parenta & & & & & & & & \\
\hline No & 375 & 47.3 & 190 & 50.7 & 185 & 49.3 & 0.34 & 0.554 \\
\hline Yes & 418 & 52.7 & 203 & 48.6 & 215 & 51.4 & & \\
\hline
\end{tabular}

*Significance level at $a=0.05$

smoked. The participants had a high frequency of consumption of dietary salt (64.4\%), greasy food (62.5\%), sweet food (50.7\%) and uncooked food (44.9\%). Five factors were found to be significantly associated with T2DM: tribe, exercise, BMI, parental history of T2DM, and triglycerides. Another four factors were found to be significantly associated with HT: sex, dietary salt intake, BMI, and parental history of HT.

The results of our study revealed very interesting information on the prevalence of T2DM among the hill tribe elderly populations in Thailand at $16.8 \%$, which is 1.75 times higher than that of the Thai population [11]. We also found significant differences in prevalence among the various tribes. Meanwhile, the prevalence of HT was $45.5 \%$, which is almost 1.6 times greater than that of the general Thai elderly population [13]. Among the participants with HT in the hill tribe elderly population, $21.9 \%$ did not know that they had HT. In taking a closer look into tribal differences, more than half of the Yao and Lisu participants had HT. This phenomenon could be attributed to the differences in culture and lifestyle among the hill tribe people, who consume alcohol and foods that are highly sweetened and salty and do not exercise regularly.

In our study, the comorbidity rate of T2DM and HT is higher than that in an Indian sample in a study of Jaya et al. [25]. However, the T2DM prevalence of our study sample is similar to that of a sample from a study conducted by Mohamed et al. [26] among the ethnic groups in northern Sudan, with a T2DM prevalence of $18.7 \%$. Dhiraj et al. [27] reported that in different tribes of the population, there were different burdens of T2DM in the sub-Himalayan region of India. This information supports the finding that the hill tribe people in 
Table 8 Classification of participants' characteristics by biomarkers

\begin{tabular}{|c|c|c|c|c|c|c|c|c|c|c|c|c|}
\hline \multirow[t]{2}{*}{ Factors } & \multicolumn{2}{|l|}{ Uric acid } & \multirow[t]{2}{*}{$x^{2}$} & \multirow[t]{2}{*}{$p$-value } & \multicolumn{2}{|l|}{ Cholesterol } & \multirow[t]{2}{*}{$x^{2}$} & \multirow[t]{2}{*}{$p$-value } & \multicolumn{2}{|l|}{ Triglyceride } & \multirow[t]{2}{*}{$x^{2}$} & \multirow[t]{2}{*}{$p$-value } \\
\hline & Normal n (\%) & High n (\%) & & & Normal n (\%) & High n (\%) & & & Normal n (\%) & High n (\%) & & \\
\hline \multicolumn{13}{|l|}{ Sex } \\
\hline Male & $246(64.4)$ & 136 (35.6) & 38.63 & $<0.001^{*}$ & $286(74.9)$ & $96(25.1)$ & 14.28 & $<0.001^{*}$ & $309(80.9)$ & 73 (19.1) & 2.44 & 0.118 \\
\hline Female & $329(83.9)$ & $63(16.1)$ & & & $244(67.4)$ & $148(32.6)$ & & & $299(76.3)$ & $93(23.7)$ & & \\
\hline \multicolumn{13}{|l|}{ Age (years) } \\
\hline $60-69$ & 311 (77.9) & $88(22.1)$ & 6.04 & $0.049^{*}$ & $261(65.4)$ & 138 (34.6) & 4.45 & $0.108^{*}$ & 303 (75.9) & $96(24.1)$ & 6.58 & $0.037^{*}$ \\
\hline $70-79$ & 197 (71.1) & $80(28.9)$ & & & 195 (78.9) & $82(21.1)$ & & & $219(79.1)$ & $58(20.9)$ & & \\
\hline$\geq 80$ & $67(68.4)$ & 31 (31.6) & & & $74(78.7)$ & $24(21.3)$ & & & $86(87.8)$ & $12(12.2)$ & & \\
\hline \multicolumn{13}{|l|}{ Tribe } \\
\hline Akha & 101 (78.3) & $28(21.7)$ & 20.19 & $0.018^{*}$ & 95 (73.6) & $34(26.4)$ & 17.05 & $0.004^{*}$ & 99 (76.7) & $30(23.3)$ & 8.86 & 0.114 \\
\hline Lahu & $113(85.0)$ & $20(15.0)$ & & & $100(75.2)$ & $33(24.8)$ & & & $96(72.2)$ & 37 (27.8) & & \\
\hline Hmong & $81(64.3)$ & $45(35.7)$ & & & $93(73.8)$ & $33(26.2)$ & & & $100(79.4)$ & 26 (20.6) & & \\
\hline Yao & $96(74.4)$ & 33 (25.6) & & & $82(90.0)$ & $47(9.1)$ & & & $98(75.9)$ & $31(24.1)$ & & \\
\hline Karen & $99(76.7)$ & $30(23.3)$ & & & $72(55.8)$ & $57(44.2)$ & & & $111(86.0)$ & $18(14.0)$ & & \\
\hline Lisu & 85 (66.4) & 43 (33.6) & & & 88 (68.8) & $40(31.2)$ & & & $104(81.3)$ & 24 (18.7) & & \\
\hline
\end{tabular}

*Significance level at $\mathrm{a}=0.05$

Thailand originate from Tibet $[14,16]$, which is close to those living in the sub-Himalayan region of India. Therefore, the T2DM and HT prevalence among the 6 hill tribes in Thailand are possibly different.

A study using a mass database in Korea reported that regular and frequent exercise led to reduced T2DM mortality and morbidly rates, particularly in the elderly population [28]. A study in Saudi Arabia also reported that sufficient physical exercise was a protective factor against T2DM development [29]. This result is similar to the finding of our study that regular exercise and highly active physical work serve as protective factors against T2DM among the hill tribe elderly populations in Thailand. Regarding BMI, Kulaya et al. [30] reported that increasing BMI was identified as a major risk factor for T2DM in the Thai population. In a study of Asian Americans in the United States, a BMI $<23$ or overweight was detected as a risk factor for T2DM development [31]. Moreover, a case-control study aimed at assessing the association between BMI and T2DM in the Mid-Atlantic region found a heavy association between increasing BMI and T2DM, after controlling for all confounding factors [32]. However, in a study among Afro-Trinidadians in the United States in 2016, no significant difference in BMI was found between those who had T2DM and those who did not [33]. In our study, it was found that increasing BMI or overweight was a risk factor for T2DM in the hill tribe elderly populations.

Many studies [34-36] have reported that having a parental or family history of diabetes or first-degree relatives with diabetes was associated with the development of T2DM, which is consistent with the findings of our study. Triglyceride levels are another factor related to the development of T2DM. A retrospective longitudinal large-scale study conducted between the year 2000 and 2012 found that every $10 \mathrm{mg} / \mathrm{dL}$ increase in triglyceride levels significantly increased the risk of T2DM by $4.0 \%$ in the United States [37]. In addition, Ming et al. [38] reported that an increase in triglycerides was a risk factor for type 2 diabetes among those living in rural China. These studies present findings similar to those of this study, such that higher triglyceride levels are a risk factor for T2DM. Different tribes or races also have significant associations with T2DM. The studies of Vitor [39] and Diego et al. [40], which were conducted in the United States using different study designs, revealed that differences in the races of parents had an impact on the development of HT in their children. However, in our study, there was no significant difference in HT prevalence among the tribes.

Jugal et al. [41] reported that there were several factors associated with HT among those living in rural Delhi, India, such as older age, alcohol use, education and cholesterol levels. However, sex was not found to be associated with HT. On the other hand, Saswata et al. [42] reported that females had a greater chance of developing HT than males in a study conducted in western India. Daily food consumption is one of the predictors for HT. Daily consumption of salty foods is one of the risk factors of HT. This finding is supported by several studies [43-45] that show that dietary salt intake was highly associated with HT development in developing and developed countries and in urban and rural areas. In this 
Table 9 Factors associated with T2DM in univariate and multivariate analyses $(n=775)^{* *}$

\begin{tabular}{|c|c|c|c|c|c|c|c|c|c|c|}
\hline \multirow[t]{3}{*}{ Factors } & \multicolumn{4}{|c|}{ T2DM } & \multirow[t]{3}{*}{ OR } & \multirow[t]{3}{*}{$95 \% \mathrm{Cl}$} & \multirow[t]{3}{*}{$p$-value } & \multirow[t]{3}{*}{$\mathrm{OR}_{\text {adj }}$} & \multirow[t]{3}{*}{$95 \% \mathrm{Cl}$} & \multirow[t]{3}{*}{$p$-value } \\
\hline & \multicolumn{2}{|l|}{ Yes } & \multicolumn{2}{|l|}{ No } & & & & & & \\
\hline & $n$ & $\%$ & $\mathrm{n}$ & $\%$ & & & & & & \\
\hline \multicolumn{11}{|l|}{ Sex } \\
\hline Mal & 66 & 17.3 & 316 & 82.7 & 1.00 & & & & & \\
\hline Female & 64 & 16.3 & 329 & 83.7 & 0.93 & $1.02-2.02$ & 0.712 & & & \\
\hline \multicolumn{11}{|l|}{ Tribe } \\
\hline Akha & 11 & 8.6 & 117 & 91.4 & 1.00 & & & 1.00 & & \\
\hline Lahu & 26 & 19.5 & 107 & 80.5 & 2.58 & $1.37-4.85$ & $0.013^{*}$ & 2.89 & $1.32-6.33$ & $0.008^{*}$ \\
\hline Hmong & 11 & 8.1 & 124 & 91.9 & 0.94 & $0.45-1.96$ & 0.896 & 0.91 & $0.35-2.31$ & 0.845 \\
\hline Yao & 26 & 21.5 & 95 & 78.5 & 2.91 & $1.54-5.48$ & $0.006^{*}$ & 3.47 & $1.58-7.62$ & $0.002^{*}$ \\
\hline Karen & 34 & 26.4 & 95 & 73.6 & 3.80 & $2.06-7.03$ & $<0.001^{*}$ & 5.03 & $2.35-10.78$ & $<0.001^{*}$ \\
\hline Lisu & 22 & 17.1 & 107 & 82.9 & 2.18 & $1.14-4.13$ & $0.046^{*}$ & 2.73 & $1.22-6.07$ & $0.014^{*}$ \\
\hline \multicolumn{11}{|l|}{ Age (year) } \\
\hline $60-69$ & 75 & 18.8 & 324 & 81.2 & 1.00 & & & & & \\
\hline $70-79$ & 39 & 14.3 & 234 & 85.7 & 0.72 & $0.50-1.02$ & 0.127 & & & \\
\hline$\geq 80$ & 16 & 15.5 & 87 & 84.5 & 0.79 & $0.48-1.30$ & 0.444 & & & \\
\hline \multicolumn{11}{|l|}{ Smoking } \\
\hline No & 78 & 16.4 & 398 & 83.6 & 1.00 & & & & & \\
\hline Ever in the past & 34 & 23.1 & 113 & 76.9 & 1.53 & $1.04-2.24$ & $0.064^{*}$ & & & \\
\hline Yes & 18 & 11.8 & 134 & 88.2 & 0.68 & $0.43-1.08$ & 0.177 & & & \\
\hline \multicolumn{11}{|l|}{ Alcohol use } \\
\hline No & 79 & 15.0 & 447 & 85.0 & 1.00 & & & & & \\
\hline Ever in the past & 26 & 19.3 & 109 & 80.7 & 1.35 & $0.89-2.03$ & 0.230 & & & \\
\hline Yes & 25 & 21.9 & 89 & 78.1 & 1.58 & $1.04-2.42$ & $0.072^{*}$ & & & \\
\hline \multicolumn{11}{|l|}{ Salty food } \\
\hline No & 151 & 53.5 & 131 & 46.5 & 1.00 & & & & & \\
\hline Yes & 266 & 52.1 & 245 & 47.9 & 0.94 & $0.70-1.26$ & 0.687 & & & \\
\hline \multicolumn{11}{|l|}{ Greasy food } \\
\hline No & 155 & 52.2 & 142 & 47.8 & 1.00 & & & & & \\
\hline Yes & 258 & 52.0 & 238 & 48.0 & 0.99 & $0.74-1.32$ & 0.962 & & & \\
\hline \multicolumn{11}{|l|}{ Sweet food } \\
\hline No & 202 & 51.7 & 189 & 48.3 & 1.00 & & & & & \\
\hline Yes & 184 & 45.8 & 218 & 54.2 & 0.78 & $0.59-1.04$ & 0.097 & & & \\
\hline \multicolumn{11}{|l|}{ Exercise } \\
\hline No & 21 & 26.6 & 58 & 73.4 & 1.00 & & & 1.00 & & \\
\hline Highly active physical work & 45 & 16.7 & 225 & 83.3 & 0.55 & $0.33-0.90$ & $0.050^{*}$ & 0.48 & $0.25-0.91$ & $0.024^{*}$ \\
\hline Yes & 64 & 15.0 & 362 & 85.0 & 0.48 & $0.30-0.78$ & $0.013^{*}$ & 0.45 & $0.24-0.83$ & $0.011^{*}$ \\
\hline \multicolumn{11}{|l|}{ BMl } \\
\hline Normal & 39 & 12.6 & 271 & 87.4 & 1.00 & & & 1.00 & & \\
\hline Underweight & 13 & 11.4 & 101 & 88.6 & 0.89 & $0.51-1.56$ & 0.743 & 0.90 & $0.45-1.80$ & 0.773 \\
\hline Overweight & 78 & 22.2 & 273 & 77.8 & 1.98 & $1.39-2.82$ & $0.001^{*}$ & 2.08 & $1.32-3.27$ & $0.001^{*}$ \\
\hline \multicolumn{11}{|l|}{ Parental history of DM } \\
\hline No & 217 & 42.1 & 298 & 57.9 & 1.00 & & & 1.00 & & \\
\hline Yes & 149 & 53.6 & 129 & 46.4 & 1.58 & $1.18-2.12$ & $0.002^{*}$ & 1.55 & $1.17-2.10$ & $0.001^{*}$ \\
\hline
\end{tabular}


Table 9 Factors associated with T2DM in univariate and multivariate analyses $(n=775)^{* *}$ (Continued)

\begin{tabular}{|c|c|c|c|c|c|c|c|c|c|c|}
\hline \multirow[t]{3}{*}{ Factors } & \multicolumn{4}{|c|}{ T2DM } & \multirow[t]{3}{*}{ OR } & \multirow[t]{3}{*}{$95 \% \mathrm{Cl}$} & \multirow[t]{3}{*}{$p$-value } & \multirow[t]{3}{*}{$\mathrm{OR}_{\mathrm{adj}}$} & \multirow[t]{3}{*}{$95 \% \mathrm{Cl}$} & \multirow[t]{3}{*}{$p$-value } \\
\hline & \multicolumn{2}{|c|}{ Yes } & \multicolumn{2}{|l|}{ No } & & & & & & \\
\hline & $n$ & $\%$ & $n$ & $\%$ & & & & & & \\
\hline \multicolumn{11}{|c|}{ Hypertension } \\
\hline No & 70 & 12.9 & 282 & 80.1 & 1.00 & & & & & \\
\hline Yes & 60 & 14.2 & 363 & 85.8 & 1.50 & $1.02-2.19$ & $0.035^{*}$ & & & \\
\hline \multicolumn{11}{|l|}{ Headache } \\
\hline No & 95 & 16.9 & 467 & 83.1 & 1.00 & & & & & \\
\hline Yes & 35 & 16.4 & 178 & 83.6 & 0.96 & $0.67-1.38$ & 0.875 & & & \\
\hline \multicolumn{11}{|l|}{ Dizziness } \\
\hline No & 86 & 15.9 & 456 & 84.1 & 1.00 & & & & & \\
\hline Yes & 44 & 18.9 & 189 & 81.1 & 1.23 & $0.88-1.72$ & 0.303 & & & \\
\hline \multicolumn{11}{|l|}{ Cholesterol } \\
\hline Normal & 90 & 17.3 & 430 & 82.7 & 1.00 & & & & & \\
\hline High & 38 & 16.1 & 198 & 83.9 & 0.91 & $0.64-1.29$ & 0.682 & & & \\
\hline \multicolumn{11}{|l|}{ Triglyceride } \\
\hline Normal & 88 & 14.9 & 504 & 85.1 & 1.00 & & & 1.00 & & \\
\hline High & 40 & 24.4 & 124 & 75.6 & 1.84 & $1.29-2.63$ & $0.004^{*}$ & 1.73 & $1.10-2.73$ & $0.017^{*}$ \\
\hline
\end{tabular}

study, we also found that dietary salt intake among the hill tribe elderly populations was a significant risk factor for HT development. Another factor related to HT is BMI. Alicja et al. [46] reported that both men and women had an increased risk of HT with increasing BMI, particularly among the elderly populations. A rural Chinese cohort study in 2016 [47] and a study in Bangladesh in 2017 [48] confirmed that the increase in BMI had a significant association with HT development. These findings coincide with those of our study, which revealed that an increase in BMI was associated with a greater odds of HT development among the hill tribe elderly populations in Thailand.

The study of Ghada et al. [49] in Egypt showed a strong association between a family history of HT and the development of HT in one's offspring. A family history has been detected as a risk factor for HT among young adults and the elderly population in several countries [50-52].

Some limitations have been identified in this study, such as misunderstanding the NPO techniques before drawing blood specimens, language, and the inability to draw blood specimens in some people. Since some targeted hill tribe villages are located far away from the city, traveling to the study setting very early in the day to collect blood specimens was sometimes not practical. Other limitations included unclear information on the research procedure and not drinking and eating food for at least 8 hours before having blood drawn. Sometimes there was no cooperation from the participants, which may have occurred because they clearly did not understand the importance of laboratory interpretations. Moreover, most hill tribe elderly adults are not educated. This finding coincides with those of studies by Apidechkul et al. [53] and Apidechul [54], who reported that a high proportion of the Akha elderly population and the Lahu people were in the illiterate group. This finding could explain participants' limited understanding of the research information and lack of cooperation with the procedure.

The researchers could not draw blood from a few participants (1.26\%) because of their individual peripheral vein characteristics. However, nobody refused to provide information and a specimen. Because this lack of data would affect the predictive statistical model (logistic regressions), these participants were excluded from the analysis to ensure the accuracy of the results. Furthermore, some participants had been diagnosed as T2DM and HT before starting the study, which could possibly impact the findings of the study, particularly their knowledge, attitudes and practices, which are common limitations of the cross-sectional study design. Concerning this point, knowledge of and attitudes toward DM and HT were not included in the prediction model. Moreover, if we look closely, only attitude is significantly different among the tribes. Additionally, the number of Lahu (excess of 3participants) and Hmong (excess of 10 participants) participants exceeded the minimum requirement for the sample size due to miscommunication between the researcher and community headman. However, these excess data did 
Table 10 Factors associated with HT in univariate and multivariate analyses

\begin{tabular}{|c|c|c|c|c|c|c|c|c|c|c|}
\hline \multirow[t]{3}{*}{ Factors } & \multicolumn{4}{|l|}{$\mathrm{HT}$} & \multirow[t]{3}{*}{ OR } & \multirow[t]{3}{*}{$95 \% \mathrm{Cl}$} & \multirow[t]{3}{*}{$p$-value } & \multirow[t]{3}{*}{$\mathrm{OR}_{\mathrm{Adj}}$} & \multirow[t]{3}{*}{$95 \% \mathrm{Cl}$} & \multirow[t]{3}{*}{$p$-value } \\
\hline & \multicolumn{2}{|l|}{ Yes } & \multicolumn{2}{|l|}{ No } & & & & & & \\
\hline & $\mathrm{n}$ & $\%$ & $\mathrm{n}$ & $\%$ & & & & & & \\
\hline \multicolumn{11}{|l|}{ Sex } \\
\hline Male & 164 & 41.7 & 229 & 58.3 & 1.00 & & & 1.00 & & \\
\hline Female & 197 & 49.3 & 203 & 50.7 & 1.35 & $1.02-1.79$ & $0.034^{*}$ & 1.29 & $1.01-1.68$ & $0.031^{*}$ \\
\hline \multicolumn{11}{|l|}{ Tribe } \\
\hline Akha & 61 & 46.9 & 69 & 53.1 & 1.00 & & & & & \\
\hline Lahu & 61 & 45.9 & 72 & 54.1 & 0.95 & $0.59-1.55$ & 0.863 & & & \\
\hline Hmong & 42 & 30.0 & 98 & 70.0 & 0.48 & $0.29-0.79$ & $0.004^{*}$ & & & \\
\hline Yao & 74 & 56.9 & 56 & 43.1 & 1.49 & $0.91-2.43$ & 0.107 & & & \\
\hline Karen & 52 & 40.0 & 78 & 60.0 & 0.75 & $0.46-1.23$ & 0.261 & & & \\
\hline Lisu & 71 & 54.6 & 59 & 45.4 & 1.36 & $0.83-2.21$ & 0.215 & & & \\
\hline \multicolumn{11}{|l|}{ Age (years) } \\
\hline $60-69$ & 173 & 42.2 & 237 & 57.8 & 1.00 & & & & & \\
\hline $70-79$ & 134 & 48.0 & 145 & 52.0 & 1.26 & 0.93-1.71 & 0.131 & & & \\
\hline$\geq 80$ & 54 & 51.9 & 50 & 48.1 & 1.48 & $0.96-2.27$ & 0.075 & & & \\
\hline \multicolumn{11}{|l|}{ Smoking } \\
\hline No & 233 & 47.9 & 253 & 52.1 & 1.00 & & & & & \\
\hline Ever in the past & 65 & 43.0 & 86 & 57.0 & 0.82 & $0.56-1.18$ & 0.293 & & & \\
\hline Yes & 63 & 40.4 & 93 & 59.6 & 0.73 & $0.51-1.06$ & 0.100 & & & \\
\hline \multicolumn{11}{|l|}{ Alcohol use } \\
\hline No & 249 & 46.3 & 289 & 53.7 & 1.00 & & & & & \\
\hline Ever in the past & 64 & 46.0 & 75 & 54.0 & 0.99 & $0.68-1.44$ & 0.960 & & & \\
\hline Yes & 48 & 41.4 & 68 & 58.6 & 0.81 & $0.54-1.23$ & 0.337 & & & \\
\hline \multicolumn{11}{|l|}{ Salty food } \\
\hline No & 138 & 48.9 & 144 & 51.1 & 1.00 & & & 1.00 & & \\
\hline Yes & 307 & 60.1 & 204 & 39.9 & 1.57 & $1.17-2.01$ & $0.002^{*}$ & 1.48 & $1.14-2.00$ & $0.001^{*}$ \\
\hline \multicolumn{11}{|l|}{ Greasy food } \\
\hline No & 136 & 45.8 & 161 & 54.2 & 1.00 & & & & & \\
\hline Yes & 241 & 48.6 & 255 & 51.4 & 1.11 & $0.83-1.49$ & 0.582 & & & \\
\hline \multicolumn{11}{|l|}{ Sweet food } \\
\hline No & 202 & 51.7 & 189 & 48.3 & 1.00 & & & & & \\
\hline Yes & 197 & 49.0 & 205 & 51.0 & 0.89 & $0.68-1.18$ & 0.454 & & & \\
\hline \multicolumn{11}{|l|}{ Regular Exercise } \\
\hline Yes & 36 & 45.0 & 44 & 55.0 & 1.00 & & & & & \\
\hline Highly active physical work & 113 & 40.5 & 166 & 59.5 & 0.83 & $0.50-1.37$ & 0.472 & & & \\
\hline No & 212 & 48.8 & 222 & 51.2 & 1.16 & $0.72-1.88$ & 0.527 & & & \\
\hline \multicolumn{11}{|l|}{ BMl } \\
\hline Normal & 112 & 35.0 & 208 & 65.0 & 1.00 & & & 1.00 & & \\
\hline Underweight & 42 & 36.2 & 74 & 63.8 & 1.05 & $0.67-1.64$ & 0.816 & 2.56 & $0.70-1.70$ & 0.696 \\
\hline Overweight & 207 & 58.0 & 150 & 42.0 & 2.56 & $1.87-3.49$ & $<0.001^{*}$ & 1.37 & $1.01-1.90$ & $<0.001^{*}$ \\
\hline \multicolumn{11}{|l|}{ Parental history of HT } \\
\hline No & 155 & 41.3 & 220 & 58.7 & 1.00 & & & 1.00 & & \\
\hline Yes & 302 & 72.2 & 116 & 27.8 & 3.69 & $2.74-4.97$ & $<0.001^{*}$ & 3.38 & $2.81-4.48$ & $<0.001^{*}$ \\
\hline
\end{tabular}


Table 10 Factors associated with HT in univariate and multivariate analyses (Continued)

\begin{tabular}{|c|c|c|c|c|c|c|c|c|c|c|}
\hline \multirow[t]{3}{*}{ Factors } & \multicolumn{4}{|l|}{$\underline{\mathrm{HT}}$} & \multirow[t]{3}{*}{ OR } & \multirow[t]{3}{*}{$95 \% \mathrm{Cl}$} & \multirow[t]{3}{*}{$p$-value } & \multirow[t]{3}{*}{$\mathrm{OR}_{\mathrm{Adj}}$} & \multirow[t]{3}{*}{$95 \% \mathrm{Cl}$} & \multirow[t]{3}{*}{$p$-value } \\
\hline & \multicolumn{2}{|l|}{ Yes } & \multicolumn{2}{|l|}{ No } & & & & & & \\
\hline & $n$ & $\%$ & $\mathrm{n}$ & $\%$ & & & & & & \\
\hline \multicolumn{11}{|c|}{ Diabetes mellitus } \\
\hline No & 282 & 43.7 & 363 & 56.3 & 1.00 & & & & & \\
\hline Yes & 70 & 53.8 & 60 & 46.2 & 1.50 & $1.02-2.19$ & $0.035^{*}$ & & & \\
\hline \multicolumn{11}{|l|}{ Headache } \\
\hline No & 249 & 43.2 & 328 & 56.8 & 1.00 & & & & & \\
\hline Yes & 112 & 51.9 & 104 & 48.1 & 1.41 & $1.03-1.94$ & $0.029^{*}$ & & & \\
\hline \multicolumn{11}{|l|}{ Dizziness } \\
\hline No & 238 & 42.8 & 318 & 57.2 & 1.00 & & & & & \\
\hline Yes & 123 & 51.9 & 114 & 48.1 & 1.44 & $1.06-1.95$ & $0.019^{*}$ & & & \\
\hline \multicolumn{11}{|c|}{ Cholesterol } \\
\hline Normal & 238 & 44.9 & 292 & 55.1 & 1.00 & & & & & \\
\hline High & 115 & 47.1 & 129 & 52.9 & 1.09 & $0.80-1.48$ & 0.564 & & & \\
\hline \multicolumn{11}{|c|}{ Triglyceride } \\
\hline Normal & 262 & 43.1 & 346 & 56.9 & 1.00 & & & & & \\
\hline High & 91 & 54.8 & 75 & 45.2 & 1.60 & $1.13-2.26$ & $0.007^{*}$ & & & \\
\hline
\end{tabular}

*Significance level at $a=0.05$

not impact the results of study but rather supported the power of the tests.

Conducting research with the hill tribe people, particularly among the elderly population, required researchers to be clearly knowledgeable about the condition before reaching them. Additionally, having research assistants who were fluent in both Thai and the local hill tribe languages was an advantage for obtaining information.

\section{Conclusions}

The hill tribe elderly populations in Thailand are living with a high burden of T2DM and HT. T2DM and HT screening programs in these populations should be implemented regularly to detect early-stage and new cases. There is an urgent need to develop proper health behavior change models to reduce BMI and the consumption of dietary salt and greasy foods among the elderly populations. Moreover, a program to encourage physical exercise is also necessary. Otherwise, Thailand must budget large amounts of money to provide care and treatment for these populations in the near future.

\section{Additional file}

Additional file 1: Hill tirb Elderly Data. (XLSX 545 kb)

\section{Acknowledgements}

The author would like to thank all the participants for kindly providing all essential information regarding the research procedures. The author is also grateful to all research assistants from the Center of Excellence for the Hill tribe Health Research for their help in data collection. The author would like to thank The National Research Council of Thailand and Mae Fah Luang University, Thailand in support the grant.

\section{Funding}

This research was supported by the National Research Council of Thailand and Mae Fah Lung University, Thailand (Grant Number 77-2015).

\section{Availability of data and materials}

The raw data supporting these findings can be found in the Additional file 1.

\section{Authors' contributions}

TA sought funding, designed the study protocols and procedures, collected data, analyzed and interpreted data, drafted, revised, and approved the final version of the manuscript.

\section{Ethics approval and consent to participate}

Consent to participate, all study instruments and procedures were approved by the Ethics Committee for Human Research, Mae Fah Laung University, Chiang Rai, Thailand (No. REH-58087). All participants received an oral and written explanation and provided their consent before a voluntary agreement was witnessed and documented by signature or fingerprint.

Competing interests

The author declares that he has no competing interests.

\section{Publisher's Note}

Springer Nature remains neutral with regard to jurisdictional claims in published maps and institutional affiliations.
BMl: Body mass index; DALYS: Disability adjusted life year; HT: Hypertension; ID: Identification; IOC: Item objective congruence; NPO: Nothing per oral; T2DM:: Type 2 diabetes mellitus; WHO: World Health Organization 


\section{Received: 1 February 2018 Accepted: 24 May 2018}

Published online: 05 June 2018

\section{References}

1. World Health Organization (WHO). Country and region data on diabetes and hypertension, 2017. 2017. http://www.who.int/diabetes/facts/world_ figures/en/index2.html

2. World Health Organization (WHO). Fact sheet: Diabetes mellitus Novembe 2017. 2017. http://www.who.int/mediacentre/factsheets/fs312/en/

3. Centers for Disease Control and Prevention (CDC). High blood pressure risk factors 2017. 2017. https://www.cdc.gov/bloodpressure/risk_factors.htm

4. Sinna PU, Anil M, Elisabeth S, Finn BL, Reimar WT. Lifestyle factors associated with type 2 diabetes and use of different glucose-lowering drugs: Crosssectional study. PLOS ONE. 2014;9(11):e111849. https://doi.org/10.1371/ journal.pone.0111849

5. Funda DY, Zeynel AO. Treatment of type 2 diabetes mellitus in the elderly. World J Diabetes. 2017:8(6):278-85.

6. Peng L, Jin-Gang Z. Blood pressure targets in the hypertensive elderly. Chin Med J. 2017;130(16):1968-72.

7. Davies A, Janet Ol, Adewale VA, Ngozi A, Emmanuel OA, Asa A, Gbolahan O. Estimating the prevalence, hospitalization and mortality from type 2 diabetes mellitus in Nigeria: a systematic review and meta analysis. BMJ Open. 2017;7:e015424. https://doi.org/10.1136/bmjopen-2016-015424.

8. Yashikin AP, Kravchenko J, Yashin Al, Sloan F. Mortality and macrovascular risk in elderly with hypertension and diabetes: effect of intensive drug therapy. American Journal of Hypertension. 2018;31(2):220-7.

9. Sophia DF, Sunny DF. Economic burden of diabetes mellitus and its socioeconomic impact on household expenditure in an urban slum area. Int J Res Med Sci. 2017 May;5(5):1808-13.

10. World Health Organization (WHO). Diabetes country profiles, 2026: Thailand diabetes. 2016. http://www.who.int/diabetes/country-profiles/tha_en.pdf.

11. Chaicharn D, Alessandra F. Diabetes management in Thailand: a literature review of the burden, cost, and outcomes. BMC Global Health. 2013;9(11) https://doi.org/10.1186/1744-8603-9-11.

12. World Health Organization (WHO). Raised blood pressure: Situation and trend. 2017. http://www.who.int/gho/ncd/risk_factors/blood_pressure_ prevalence text/en/.

13. World Health Organization (WHO)-Thailand. Technical consultation to strengthen hypertension care in Thailand, 2017. 2017. http://www.searo. who.int/thailand/news/htn-meeting-report-draft24nov.pdf?ua=1

14. Princess Maha Chakri Siridhorn Anthropology center. Hill tribe. 2018. http://www.sac.or.th/main/index.php

15. The hill tribe welfare and development center, Chiang Rai province. Hill tribe population. The hill tribe welfare and development center. Chiang Rai: Ministry of Interior; 2017. p. 22-31.

16. Tawatchai A. A 20-year retrospective cohort study of TB infection among the hill tribe HIV/AIDS populations, Thailand. BMC Infectious Disease. 2016 16:72. https://doi.org/10.1186/s12879-016-1407-4

17. Tawatchai A, Onn L, Sirinan S. Inequity in accessing health care service in Thailand in 2015: A case study of the hill tribe people in Mae Fah Luang district, Chiang Rai, Thailand. J Health Res. 2016;30(1):67-71.

18. Sudkhed D, Tawatchai A, Rachanee S, Amornrat A, Onnalin S, Bukhari P, et al. Patterns and perception of alcohol drinking among the Lahu people, northern Thailand. J Health Res. 2018;32(3):67-71.

19. Incidence and risk factors for type 2 diabetes mellitus in transitional Thailand: results from the Thai cohort study. BMJ Open. 2016;6:e014102. https://doi.org/10.1136/bmjopen-2016-014102

20. World Health Organization (WHO). Definition and diagnosis of diabetes mellitus and intermediate hyperglycemia. 2017. http://www.who.int/ diabetes/publications/Definition\%20and\%20diagnosis\%20of\%20diabetes_ new.pdf

21. The hill tribe welfare and development center, Chiang Rai province. Hill tribe population. In: The hill tribe welfare and development center. Chiang Rai: Ministry of Interior; 2015. p. 16-27.

22. American College of Cardiolog. 2017 Guideline for prevention, detection, evaluation, and management of high blood pressure in adult. file://Users/macbookpro13/Downloads/Guidelines_Made_Simple_ 2017_HBP.pdf

23. World Health Organization (WHO). BMI classification in Asian population. 2015. http://apps.who.int/bmi/index.jsp?introPage=intro_3.html
24. American Association of Clinical Endocrinologists and American College of Endocrinology. Guidelines for management of dyslipdemia and prevention of cardiovascular disease. 2017. https://www.aace.com/files/ lipid-guidelines.pdf

25. Jaya PT, Thakur JS, Gursimer J, Sanjay J. Prevalence and determinants of comorbidity diabetes and hypertension: evidence from non communicable disease risk factor STEPS survey, India. Diabetes \& Metabolic Syndrome: Clinical Research and Reviews. 2017;11(Sup1):S459-65.

26. Mohamed AE, Abubakr BM, Hind EE, Kamal Y, Sufian KN, Waldie ME, et al. Increasing prevalence of type 2 diabetes mellitus and impact of ethnicity in north Sudan. Diabetes Research and Clinical Practice. 2018;136:93-9.

27. Dhiraj K, Ashok KB, Dinesh K, Sunil KR. Prevalence of diabetes mellitus and its risk factors among permanently settled tribal individuals in tribal and urban areas in northern state of Sub-Himalayan Region of India. International of Chronic Diseases. 2014; Article ID 380597, 9 pages. https:// doi.org/10.1155/2014/380597.

28. Woo YS, Taehee L, Da-Hye J, Hyeon CK. Diabetes, frequency of exercise, and mortality over 12 years: Analysis of the National Health Insurance ServiceHealth Screening (NHIS-HEALS) Database. J Korean Med Sci. 2018;33(8):e60.

29. Alzaheb RA, Altemani AH. The prevalence and determinants of poor glycemic control among adults with type 2 diabetes mellitus in Saudi Arabia. Diabetes Metab Syndr Obes. 2018;11:15-21.

30. Kulaya N, Natkamol C, Panuwat $P$, Jariya $H$. Screening high-risk populations for hypertension and type 2 diabetes among Thais. WHO South-East Asia Journal of Public Health. 2012;1(3):320-9.

31. Karen MK, Keith TC, Esme FT. Diabetes among Asian Americans with BMI less than or equal to 23. Diabetes\& Metabolic Syndrome: Clinical Research \& Reviews. 2017; https://doi.org/10.1016/j.dsx.2017.12.011

32. Michael LG, Neil W, Qian L, Veronica A, Jakob L, Mette H. The association of body mass index with the risk of type 2 diabetes: a case-control study nested in an electronic health records system in the United States. BMC Diabetology \& Metabolic Syndrome. 2014;6:50. https://doi.org/10.1186/1758-5996-6-50

33. Shivananda N, Kheleel S, Shivang S, Vashista S, Rukaiya S, Safayah S, et al. Investing the link between benign prostatic hypertrophy, BMl, and type 2 diabetes mellitus. Diabetes\& Metabolic Syndrome: Clinical Research Reviews. 2017:11(Siup2):S627-30.

34. Deepark KD, Arun KC, Mamta S, Swayamsidha M, Binoy KM, Anoj KB. Effect of parental history of diabetes on markers of inflammation insulin resistance and atherosclerosis in first degree relatives of patients with type 2 diabetes mellitus. Diabetes \& Metabolic Syndrome: Clinical Research \& Reviews. 2017; https://doi.org/10.1016/j.dsx.2017.12.004

35. Kara MG, Shannon KC, Duck-chul L, Xuemei SS, Steven PH, Steven NB. Association of cardiorespiratory fitness and parental history of diabetes with risk of type 2 diabetes. Diabetes research and Clinical Practice. 2012;95(3):425-31.

36. Chaochen W, Hiroshi Y, Koji T, Hideaki T, Keiko W, Yuanying L, et al. Association between parental history of diabetes and the incidence of type 2 diabetes mellitus differs according to the sex of the parent and offspring's body weight: A finding from a Japanese worksite based cohort study. Preventive Medicine. 2015;81:49-53.

37. Aman B, Eytan C, Elad G, Pearl L, Moshe G, llan K. Triglyceride levels and risk of type 2 diabetes mellitus: A longitudinal large study. J Investig Med. 2016; 64(2):383-7.

38. Ming Z, Bingyuan W, Yu L, Xizhuo S, Xinping L, Chongjian W, et al. Cumulative increased risk of incident type 2 diabetes mellitus with increasing triglyceride glucose index in normal-weight people: the rural Chinese cohort. BMC Cardiovascular Diabetology. 2017;16(30): https://doi. org/10.1186/s12933-017-0514-X

39. Victor $G$. Pregravid hypertension may have different secondary sex ratio effects in different races in the United States. International Journal of Cardiology. 2017:249:333-5.

40. Diego GD, Arthur EM, Raphael MR, Romulo AF, Bruna TC, Mariana RP, et al. Association between hypertension in adolescents and the health risk factors of their parents: an epidemiological family study. Journal of American Society of Hypertension. 2018; https://doi.org/10.1016/j.jash. 2017.12.011

41. Jugal K, Neeru G, Charu K, Neeta K. Prevalence of hypertension and determination of its risk factors in rural Delhi. International Journal of Hypertension. 2016; https://doi.org/10.1155/2016/7962595.

42. Saswata G, Simantini M, Anamitra B. Sex difference in the risk profile of hypertension: A cross-sectional study. BMJ open. 2016:e010085. https://doi. org/10.1136/bmjopen-2015-010085. 
43. Sung KH. Dietary salt intake and hypertension. Electrolyte Blood Press. 2014; 12(1):7-18.

44. William BF, David GE, Claudine TJ, William SW. Dietary sodium and health: more than just blood pressure. J Am Coll Cardiol. 2015;65(10):1042-50

45. Mandapaka RT, Rachabathuni S. Prevalence of hypertension and its relationship between dietary salt intake in urban population. J Community Med Health. 6:426. https://doi.org/10.4172/2161-0711.1000426.

46. Alicja S, Anna L. BMI, hypertension and low bone mineral density in adult men and women. HOMO-Journal of Comparatie Human Biology. 2012;63(4):282-91.

47. Ming Z, Yang Z, Haohang S, Xinping L, Chongjian W, Linlin L. Effect of dynamic change in body mass index on the risk of hypertension: results from the rural Chinese chohort study. International Journal of Cardiology. 2017;238:117-22

48. Mohammed MR, Shamima A, Jenny J, Mohammed SR, Papia S. Trend, projection, and appropriate body mass index cut-off point for diabetes and hypertension in Bangladesh. Diabetes Research and Clinical Practice. 2017; 126:43-53.

49. Ghada Y, Ibrahim ET, Dina O, Ahmed S, Essam B, Zeinab A, et al. Familial history of hypertension as a predictor of increased arterial stiffness in normotensive offspring. The Egypt Heart Journal. 2017;69(1):37-44.

50. Menghetti E, Strisciuglio P, Spagnolo A, Carletti M, Paciotti G, Muzzi G, et al. Hypertension and obesity in Italian school children: The role of diet, life style and family history. Nutrition, metabolism and Cardiovascular Diseases. 2015;25(6):602-7.

51. Dorairaj P, Panniyammakal J, Shreeparna G, Roopa S, Vamadevan SA, Dimple $K$, et al. Prevalence and incidence of hypertension: results from a representative cohort of over 16,000 adults in three cities of South Asia. Indian Heart Journal. 2017;69(4):434-41.

52. Mercedes RC, Guadalupe XA, Shrikant IB. Association of cardiovascular risk factors between Hispanic/Latino parents and youth: the Hispanic community health study/study of Latino youth. Annals of Epidemiology. 2017;27(4):260-8.

53. Tawatchai A, Pilasinee W, Siriyaporn S, Thapakorn R. Health situation of Akha hill tribe in Chiang Rai province, Thailand. Journal of Public Health and Development. 2016;14(1):77-97.

54. Tawatchai A. Prevalence of thalassemia carriers among the Lahu hill tribe population, Chiang Rai, Thailand. Asian Biomedicine. 2015;9(4):527-33.

\section{Ready to submit your research? Choose BMC and benefit from:}

- fast, convenient online submission

- thorough peer review by experienced researchers in your field

- rapid publication on acceptance

- support for research data, including large and complex data types

- gold Open Access which fosters wider collaboration and increased citations

- maximum visibility for your research: over $100 \mathrm{M}$ website views per year

At BMC, research is always in progress.

Learn more biomedcentral.com/submissions 